\title{
Coping with Interoperability in the Development of A Federated Research Infrastructure: Achievements, Challenges and Recommendations from the JA Infact
}

\section{Juan González-García}

Aragon Health Sciences Institute: Instituto Aragones de Ciencias de la Salud

\section{Francisco Estupiñán-Romero}

Aragon Health Sciences Institute: Instituto Aragones de Ciencias de la Salud Javier González-Galindo

Aragon Health Sciences Institute: Instituto Aragones de Ciencias de la Salud

Carlos Telleria-Orriols

Aragon Health Sciences Institute: Instituto Aragones de Ciencias de la Salud

\section{Luigi Palmieri}

Istituto Superiore di Sanità: ISS

\section{Andrea Faragalli}

Università Politecnica delle Marche: Universita Politecnica delle Marche

\section{Ivan Pristās}

Croatian Institute of Public Health: Hrvatski zavod za javno zdravstvo

\section{Jakov Vuković}

Croatian Institute of Public Health: Hrvatski zavod za javno zdravstvo

\section{Jānis Misinš}

Centre for Disease Prevention and Control, Latvia

Irisa Zile

Centre for Disease Prevention and Control, Latvia

Enrique Bernal-Delgado ( $\nabla$ ebernal.iacs@aragon.es )

Institute for Health Sciences in Aragon (IACS) https://orcid.org/0000-0002-0961-3298

InfAct Consortium

Sciensano

\section{Methodology}

Keywords: Population health research, Health data, Secondary use of data, Federated research infrastructures, European Interoperability Framework, Legal Interoperability, Organizational 
Interoperability, Semantic Interoperability, Technological Interoperability, Federated learning

Posted Date: July 1st, 2021

DOI: https://doi.org/10.21203/rs.3.rs-632073/v1

License: (c) (1) This work is licensed under a Creative Commons Attribution 4.0 International License. Read Full License

Version of Record: A version of this preprint was published at Archives of Public Health on December 1st, 2021. See the published version at https://doi.org/10.1186/s13690-021-00731-z. 


\section{Abstract}

Background: Information for Action is a Joint Action (JA InfAct on Health Information) promoted by the EU Member States and funded by the European Commission within the Third EU Health Programme (2014-2020) to create and develop solid sustainable infrastructure on EU health information. The main objective of this the JA-InfAct is to build an EU health information system infrastructure and strengthen its core elements by a) establishing a sustainable research infrastructure to support population health and health system performance assessment, b) strengthening the European health information and knowledge bases, as well as health information research capacities to reduce health information inequalities, and c) supporting health information interoperability and innovative health information tools and data sources.

Method: Follsowing a federated analysis approach, JA-InfAct developed an ad hoc federated infrastructure based on distributing a well-defined process-mining analysis methodology to be deployed at each participating partners' systems to reproduce the analysis and pooling the aggregated results from the analyses. To overcome the legal interoperability issues on international data sharing, data linkage and management, partners (EU regions) participating in the cases study worked coordinately to query their real-world healthcare data sources complying with a common data model, executed the process mining analysis pipeline on their premises, and shared the analysis results enabling international comparison and the identification of best practices on stroke care.

Results: the ad hoc federated analysis infrastructure was designed and built upon open source technologies providing partners with the capacity to exploit their data and generate stroke care pathway analysis dashboards. These dashboards can be shared among the participating partners or to a coordination hub without legal issues enabling the comparative evaluation of the caregiving activities for acute stroke across regions.

Nonetheless, the approach is not free of a number of challenges that have been solved, and new challenges that should be addressed in the eventual case of scaling up. For that eventual case, 12 recommendations on the different layers of interoperability have been provided.

Conclusion: The JA InfAct federated analysis infrastructure has been able to cope with all levels of the interoperability -legal, organisational semantic and technological. Any proposal for extension would require upgrade so as to deal with new challenges on interoperability such as large scale application of GDPR principles, developing high-profile resources capacity across Europe, developing a common strategy to data quality assurance and preparedness for high performance computing and federated learning.

\section{Background}

"Information for Action!" is a Joint Action (JA-InfAct) on Health Information promoted by the EU Member States and funded by the European Commission within the Third EU Health Programme (2014-2020), 
including 40 partners in $28 \mathrm{EU}$ and associated countries. The main aim of this the JA-InfAct is to build an EU health information system infrastructure and strengthen its core elements by a) establishing a sustainable research infrastructure to support population health and health system performance assessment, b) strengthening the European health information and knowledge bases, as well as health information research capacities to reduce health information inequalities, and c) supporting health information interoperability and innovative health information tools and data sources. One of the underlying tasks has been setting up the pillars for the design, preparation and implementation of a federated research infrastructure that leverages the use of health data to carry out policy-oriented research.

Paramount in the development of a federated research infrastructure (FRI), where data is leveraged from multiple and heterogeneous data sources hosted in multiple sites with different governance models, is interoperability. JA-InfAct interoperability elements are framed in the recommendations report by the European Interoperability Framework (EIF) [1]. According to the EIF, interoperability is defined as "the ability of organisations to interact towards mutually beneficial goals, involving the sharing of information and knowledge between these organisations, through the business processes they support, by means of the exchange of data between their ICT systems". In the specific case of JA InfAct, interoperability refers to the capacity to capture coherent data in the different partners, being able to reproduce the same analyses and being capable of sharing the results of these analyses. The design, development and implementation of the JA InfAct federated research infrastructure have built upon the interoperability layers introduced by the EIF and depicted in Figure 1 [1]. The EIF framework classifies the interoperability elements in four layers that need to be addressed for a successful interoperable (public) service -namely Legal, Organizational, Semantic and Technical interoperability.

This article seeks to describe the process and challenges followed in the context of JA InfAct to cope with the different layers of interoperability when trying to answer population health research queries in the context of a federated infrastructure. Likewise, the report provides recommendations for the eventual reallife implementation of such a federated infrastructure.

\section{Methods}

InfAct has addressed the different interoperability challenges to build an FRI, following an approach based on case studies (also known as pilots). In Table 1, there is a synthesis of the three use cases deployed along the Joint Action. The selection had mainly to do different levels of maturity - from the simplest definition of a study and how materialized into the required documentation (ie, case study on dementia), the definition of a population based indicator that materializes in a SQL query that is distributed to elaborate indicators in house (ie., case study on resilient populations), to a full distribution exercise where a common data model is built, an analytical pipeline developed to be interoperable is distributed among a number nodes and the nodes run the analyses and produce the expected research outputs that are sent back to a coordination hub (i.e., case study on stroke care pathways). As it will be 
detailed, the latter has provided full insight on how to make interoperability a reality in the context of JA InfAct federated infrastructure.

In short, the JA-InfAct federated analysis infrastructure is an ad hoc infrastructure solution proposed to perform a cross-border analysis. The core element of the analysis infrastructure is the process-mining methodology, where real-world datasets are combined and then processed to generate the care pathways process models within the premises of each participant partner.

The analysis methodology is encapsulated in a software distribution solution that acts as the central element of the federated analysis infrastructure that leverages the data capture and execution of the analyses across different partners and the exchange of the results. All the methodologies and solutions are designed and implemented following a security-and privacy-by-design approaches and to fulfil the interoperability challenge, i.e., how to work with different information systems, data sets and software solutions existing in each of the participating partners with a complete coherence of the analysis results.

This Section is organised to describe these three elements, using as a case study the empirical discovery of care pathways of acute ischemic stroke. Subsection 2.1 summarises the process-mining analysis, further commented in [2]. Subsection 2.2 details the technical aspects of the federated analysis infrastructure, considering the software elements and the orchestration elements to reach the desired solutions. Subsection 2.3 focuses on all the interoperability elements that have been considered within the first two elements (the analysis methodology and the analysis infrastructure) and specific organisational agreements reached between partners for a successful deployment of such an infrastructure. 
Table 1

JA-InfAct use cases

\begin{tabular}{|c|c|c|c|c|c|}
\hline Use case & Purpose & $\begin{array}{l}\text { Data } \\
\text { sources }\end{array}$ & $\begin{array}{l}\text { Common } \\
\text { Data model } \\
\text { (main entities) }\end{array}$ & Distribution & Hubs \\
\hline $\begin{array}{l}\text { Dementia } \\
\text { care }\end{array}$ & $\begin{array}{l}\text { Identification of } \\
1 \text {-year follow up } \\
\text { contacts and } \\
\text { associated } \\
\text { costs }\end{array}$ & $\begin{array}{l}\begin{array}{l}\text { Insurance } \\
\text { data }\end{array} \\
\text { PC EHR } \\
\text { Hospital } \\
\text { stays } \\
\text { Prescriptions } \\
\text { ER data } \\
\text { RHB } \\
\text { contacts } \\
\text { Billing data }\end{array}$ & $\begin{array}{l}\text { Individual patient } \\
\text { Care provider Point of } \\
\text { care }\end{array}$ & $\begin{array}{l}\text { Data model } \\
\text { specification } \\
\text { (v0.1) }\end{array}$ & $\begin{array}{l}\text { Aragon } \\
\text { (ES) } \\
\text { France } \\
\text { (FR) }\end{array}$ \\
\hline $\begin{array}{l}\text { Desirable } \\
\text { health } \\
\text { services } \\
\text { utilization }\end{array}$ & $\begin{array}{l}\text { Elaboration of a } \\
\text { population- } \\
\text { based health } \\
\text { indicator based } \\
\text { on those lower } \\
\text { users }\end{array}$ & $\begin{array}{l}\text { Insurance } \\
\text { data } \\
\text { PC EHR } \\
\text { Prescriptions } \\
\text { Hospital } \\
\text { stays }\end{array}$ & $\begin{array}{l}\text { Individual Insurees } \\
\text { Residence }\end{array}$ & $\begin{array}{l}\text { Protocol, } \\
\text { data model } \\
\text { specification } \\
\text { and SQL script } \\
\text { for data } \\
\text { transformation } \\
\text { (v1.0) }\end{array}$ & $\begin{array}{l}\text { Wales } \\
\text { NHS } \\
\text { (UK) } \\
\text { Aragon } \\
\text { (ES) }\end{array}$ \\
\hline $\begin{array}{l}\text { Stroke } \\
\text { care } \\
\text { pathway }\end{array}$ & $\begin{array}{l}\text { Discovery of the } \\
\text { actual care } \\
\text { pathway for } \\
\text { Acute Stroke } \\
\text { patients }\end{array}$ & $\begin{array}{l}\text { Insurance } \\
\text { data } \\
\text { ER data } \\
\text { Hospital } \\
\text { data }\end{array}$ & $\begin{array}{l}\text { Individual patient } \\
\text { Care provider } \\
\text { Residence/Healthcare } \\
\text { area } \\
\text { Episodes } \\
\text { Events }\end{array}$ & $\begin{array}{l}\text { Complete } \\
\text { solution: } \\
\text { Docker with } \\
\text { open source } \\
\text { Log builder } \\
\text { and } \\
\text { Process } \\
\text { Mining } \\
\text { FAlR } \\
\text { publication } \\
\text { (v14.0) }\end{array}$ & $\begin{array}{l}\text { Aragon } \\
\text { (ES) } \\
\text { Marche } \\
\text { (IT) } \\
\text { HU } \\
\text { Zagreb } \\
\text { (HR) } \\
\text { HU } \\
\text { Riga } \\
\text { (LV) }\end{array}$ \\
\hline
\end{tabular}

Description of the case study: process-mining based analysis methodology 
The process-mining methodology used in the JA-InfAct has been previously introduced for a similar use case at a regional level in the work by González-García et al. [2]. It is based on analysing real-world datasets related to stroke care using process mining techniques [3], [4], and, specifically, process discovery. The analysis aims to detect how theoretical pathways of care or clinical guidelines, such as the acute ischemic stroke care pathway defined in [5], are implemented in real life.

The methodology is illustrated in Fig. 2 and comprises four main elements: 1) capture the real-world data to be further processed, considering the start of the acute stroke care pathway should be captured in the urgent care (i.e. accident and emergency care services) and hospital care information systems and the patients information database should contain specific details on patients characteristics; 2 ) transform the data from their specific information systems into a defined common data model, which contains the actual semantics of the contents in the form of different entities (i.e., patients, visits, procedures, etc.), the variables that define entities (i.e., age or sex for patients, visit date and hospital, or procedure date and code), their relationships (i.e., when a patient visited a hospital where he or she received a procedure) along with the encoding systems prevalent in the different nodes (ie, ICD 9th, ICD 10th) ; 3 ) process the data stored in the common data model to generate an event log (where each register in the data set represents an activity and its attributes), using the Event Log Buildertool that feed the final Process Mining Pipeline tool, which generates the empirical process models; and 4) compare and contrast the process models obtained to verify the actual care pathways in the different countries. The outputs of the methodology are the process models that can be depicted, for example, as process maps that present the real-life transitions between events, the number of patients along each trajectory and throughput times (see later in the results section).

\subsection{Description of the federated infrastructure}

The JA-InfAct federated analysis infrastructure has been designed with the objective of distributing the analytical pipelines using the data of the different partners, in this case study replicating the process mining methodology without requiring any individual data movement from the partners to other partners or to a Coordination Hub. The Coordination Hub, in this context, is responsible for developing the analysis scripts, supporting each participant partner in their deployment and producing insights from the comparison between partners' outputs. The term federated infrastructure is used because participating partners can act independently without requiring the rest of the partners to perform the analyses.

Figure 3 presents the schema of the distribution workflow. The partners involved in the infrastructure are generally considered Data Hubs in the Figure. They are responsible for transforming and loading their data sets in the common data model format previously defined and agreed on. As stated initially, this is an ad hoc infrastructure, which means that the code distribution is actually done in two steps: first, the Coordination Hub encapsulates the process mining analysis scripts into a portable execution environment and, second, each Data Hub gets this portable execution environment, deploys it and runs it in their premises, indicating where the data is located. 
The analysis process starts, point 1 in the Figure, when the Coordination Hub distributes the process mining analysis scripts among the Data Hubs. Next, in point 2 of the Figure, partners fetch the scripts into their systems, indicate the input data placement to the analysis code and run the analyses. As a result of this point, each partner obtains their own results (ie, stroke care pathways). Finally, depicted as point 3 of the Figure, partners send back their results to the Coordination Hub. This feedback in the form of local results is sent in this case manually, by compressing the output dashboard and mailing it to a specific address. Finally, once the Coordination Hub has gathered information from all partners, it compares them to present a final analysis, point 4 in the Figure.

Note that this architecture follows the privacy-by-design principle as all data is governed under the legal provisions of the hosting institutions and no individual data is moved outside partner premises tackling various relevant legal interoperability issues raising legal barriers to cross-border data sharing. Results and data that feeds back from partners to the Coordinator Hub are always outputs, in this case, aggregated measures in the form of the process models, and runtime application error logs. In addition, following the secure-by-design principle and as a way to build trust among federated infrastructure participants, it is important to remark that all the analysis and deployment solutions presented are open source, thus all source code of the scripts for data management and analysis are auditable by the participants, ensuring the credibility on what is going to be executed, enhancing the reliability of the results and helping to increase the solution quality through the contribution from the partners.

\section{Interoperability layers}

What has been described in the two previous subsections relies on guaranteeing the interoperability among the different components of the solution, as well as the relationship within the involved partners in terms of trust, governance and legal compliance. In the following subsections we describe how the different layers of the EIF have been achieved in the context of the JA InfAct federated research infrastructure.

\section{Legal interoperability. The General Data Protection Regulation (GDPR)}

The top layer in the EIF interoperability model is legal interoperability. Given the compliance with GDPR and overarching Ethical principles, legal interoperability is about "ensuring that organisations operating under different legal frameworks, policies and strategies are able to work together".

Being the objective of this federated infrastructure to analyse care pathways using patients' data, the legal framework to be considered are those regulating the use of personal health data for research purposes. There has been a huge effort by European legislators in this topic so as to homogenize the use of this kind of data across the EU Member States, materialised in the General Data Protection Regulation (GDPR) [6] the EU law that establishes the conditions for the legal interoperability of the JA-InfAct federated infrastructure. 
The second layer to be discussed is the organisational interoperability layer. Organisational interoperability refers "to the way in which public administrations align their business processes, responsibilities and expectations to achieve commonly agreed and mutually beneficial goals".

The JA-InfAct federated analysis infrastructure was designed as a client-server infrastructure, with a coordination hub orchestrating the whole process (consolidation of the data model, development of the analytical pipeline including technological solutions) and a node counterpart where a contact person performed the following duties: 1 ) detect the staff with the knowledge to perform the required tasks; 2 ) assist to the work meetings to ensure the proper coordination of the work; 3) commit the necessary resources to develop the work; 4) provide the required feedback to improve and solve the possible issues that appear during the development; and 5) carry on the analyses and feedback and interpret the results considering the local context.

Semantic interoperability. The common data model and the data codifications.

The semantic interoperability ensures that "the precise format and meaning of exchanged data and information is preserved and understood throughout exchanges between parties". So, in practice, it is required to guarantee that when performing the care pathway analyses in the different partners the data and the results refer to the very same caregiving processes.

The cornerstone element in the semantic interoperability layer is the common data model (CDM), in which the data entities and their relationships are defined, and serves as the common storage to perform further analyses. The CDM design was iteratively refined to express the actual caregiving settings in the different partners. For example, initial versions of the fibrinolysis treatment and the thrombectomy procedure, key for a rapid response on ischaemic strokes, were not considered to appear within urgent care events, while in further refinements these activities were included in this setting. At the moment of writing this paper, the CDM was on its 14 th revision.

It is important to note that, as a fundamental component of the CDM apart from the entities and relationships, it is also defined how the information is codified within the data model variables; thus, which are the encoding systems or standards used in the different nodes of the federation.

\section{Technical interoperability. The Event Log Build and the Process Mining Pipeline}

The technical interoperability layer covers "the applications and infrastructures linking systems and services. Aspects of technical interoperability include interface specifications, interconnection services, data integration services, data presentation and exchange, and secure communication protocols".

The most important element in the technical interoperability layer of the JA-InfAct federated analysis infrastructure is the "package deployment system", i.e., how the different source code of the analysis 
scripts is encapsulated so as to be easily transmitted from the Coordinator Hub to the partners, easily executed in the partners to perform the analysis in their premises and, the results, easily transmittable back to the Coordination Hub.

As a final point regarding the technical interoperability, it is important to highlight that the analysis code distributed to the partners relies on having the input data of each partner in the CDM format. It is the responsibility of each partner to create the extraction, transformation and load (ETL) processes that capture the required data in their health information systems and fill the CDM according to the agreed definitions.

\section{Results}

The JA InfAct federated infrastructure, as designed, has been able to yield the intended output in the four nodes composing the case study on stroke -each node has been able to produce a dashboard depicting the real-life care pathways in the four countries. This section details the actual implementation of the process mining methodology shown in Figure 1 within the JA-InfAct FRI. Therefore, it includes the specification of the common data model, distribution of the analytical pipeline, implementation of the pipeline in the different nodes and the collection of the outputs.

\section{Specification of the data model}

The research question sent out to the federation materialized in: 1) a definition of the cohort of patients as those who, in the period of study, had been admitted with symptoms of stroke in an emergency ward or had a hospital admission due to stroke; 2 ) because of the different theoretical pathways, a classification of patients as confirmed ischemic stroke or hemorrhagic stroke patients; and, 3) the definition of the different activities (namely, events) that patients would follow in their journey from admission to discharge (Table 2)

As a second step, coding experts and neurologists among different partners were consulted to select the codes that conceptually better fitted the cohort definition. In our case, and for the nodes included in the exercise, we just needed to map between ICD-9th [7] and ICD-10th [8] codes.

Table 2 Types of activities considered in the process mining analysis 


\begin{tabular}{|ll|}
\hline Activity Name & Activity Description \\
\hline ER Admission & Administrative admission to Emergency Room Department \\
\hline ER First Attention & First contact with an MD in the ER Department \\
\hline ER CT & Computed Tomography Scan imaging at ER Department \\
\hline ER Fibrinolysis & Fibrinolysis infusion at ER Department \\
\hline ER Thrombectomy & Thrombectomy surgical procedure at ER Department \\
\hline ER Observation Room & Observation room stay at ER Department \\
\hline ER Discharge & ER Department administrative discharge \\
\hline ER Exit & ER Department physical exit \\
\hline Hospital Admission & Hospital administrative admission \\
\hline Hospital Fibrinolysis & Fibrinolysis infusion during hospitalisation \\
\hline Hospital Thrombectomy & Thrombectomy surgical procedure during hospitalisation \\
\hline Hospital Discharge & Hospital administrative discharge \\
\hline Long-stay Hospital Admission & Long-stay (recovery) hospital administrative admission \\
\hline Long-stay Hospital Discharge & Long-stay (recovery) hospital administrative discharge \\
\hline
\end{tabular}

Once the cohort of patients was defined and the potential activities were identified and discussed, the data model was iteratively refined to express the actual caregiving settings in the different partners, so as to get a CDM. For example, initial versions of the fibrinolysis treatment and the thrombectomy procedure, key for a rapid response on ischaemic strokes, were not considered to appear within urgent care events, while in further refinements these activities were included in this setting. At the moment of writing this paper, the CDM was on its $14^{\text {th }}$ revision.

As a final result, the common data model that supported the development of this case study, comprised five main entities: patient, care provider, residence/healthcare area, episodes and events (activities). The data model is shown in Figure 4 using the Unified Model Language ${ }^{[1]}$ conceptual model notation.

The CDM for this case study can be found here http://doi.org/10.5281/zenodo.4879504

\section{Analytical pipeline}

Once the data model was common to all the nodes in the federation, the Coordination Hub developed and distributed the analytical pipeline; in this case, distributing two main pieces: a) the Event Log Builder, Python script; and, b) the Process Mining Pipeline script, written in R. 
The coordination hub packaged both pieces of software using Docker [9]. Docker is a compendium of existing Linux-based technologies that, in simple terms, is able to create isolated execution environments where software developers can guarantee that: 1) will be exactly the same wherever they are executed; 2 ) code dependencies are easily managed; and 3) the deployment of the execution environments in different locations (in the current, case, the different partner premises) is transparently managed. The first point is provided by using operating system containerization; the second point is provided by having a (nearly) infinite set packages for existing code libraries, and the third point is provided by having an environment exchange hub solution, publicly under namely the Docker Hub or privately deployed by software developers on their premises, where the execution environments are uploaded by their authors and easily downloaded by the users. For the purposes of this case study we adapted the Docker solution used in another project available at https://hub.docker.com/repository/docker/iacsbiocomputing/ictusnet_analysis.

\section{Implementation of the pipeline and production of research outputs}

The main tangible outcome of the JA-InfAct federated infrastructure are a number of interactive dashboards generated on each partner premises on the actual care pathways followed by patients with a suspicious stroke in Marche (IT), Riga (LV), Zagreb (HR) and Aragon (ES).

The interactive dashboards contain the sequence of activities followed by the patients in the cohort in each of the sites (namely, process traces in Figure 5). Next, it contains the process map with the amount of patients moving throughout the care pathway (Figure 6) and the throughput times among activities (Figure 7); and, finally, it also contains the precedence matrix, a presentation of the information present in the process map with frequency information (frequency of transitions between caregiving activities analyses) in a matrix form (Figure 8). Once the information has been gathered in the Coordination Hub, the effort done in the interoperability elements guarantees the comparability of the results.

Once the different nodes have been able to implement the analytical pipeline and produce the aforementioned outputs, dashboards are sent back to the Coordination hub for further analyses. The different dashboards, with outputs for all patients, ischaemic strokes and hemorrhagic strokes can be consulted here http://doi.org/10.5281/zenodo.4925733

\section{Discussion}

Having completed the exercise we have demonstrated the feasibility of the JA InfAct approach to federated analyses. All the layers of interoperability have been successfully considered in this case study. The exercise has provided meaningful insight into the difficulties of the implementation of such a federated infrastructure and has shed light on the ways forward. In the following section, we specifically address the challenges faced, also providing recommendations on how to tackle them in the eventual development of a federated research infrastructure.

\section{Challenges on legal interoperability}


In the JA-InfAct federated analysis infrastructure, the GDPR compliance has been materialized avoiding the use of personal data during the analysis, minimising the data requirements to complete the case study and providing reliable procedures on how to manage the data at the participant partner's premises within their governance and security assurance procedures. All these concerns are captured in the common data model and how it is filled and processed. The common data model and the data processing designed in [2] had the following characteristics to ensure GDPR compliance, especially those related to Art.5, related to personal data processing and Art.89, which regulates the research uses of personal data: 1) the data has been only used for the agreed analyses between partners ('purpose limitation); 2) the data gathered has been limited to only those variables required for the analyses and the analysis time-frame ('data minimisation'); 3) the personal data has been stored using pseudonyms to avoid possible reidentification of patients ('confidentiality'), and 4) patients' data is only stored on the premises of the partners which has the mandate/responsibility of curating such original datasets ('storage limitation').

\section{Challenges on organizational interoperability}

Organisational Interoperability is rooted in the willingness of the Data Hubs to participate and cooperatively respond to a research question that is relevant to the participants. It is labour-intensive and requires almost continuous communication between all the partners and the coordination hub and building a trust relationship based on the adjustment of use case specifications considering all inputs from participant partners, reaching consensus on each step of the process, allocating task to staff with relevant skills in each organisation through the different steps in the process and being completely transparent in all developments aimed at producing a full reproducible implementation of the analytical pipeline.

The JA InfAct federated infrastructure has been designed as a client-server infrastructure with a Coordination ub that orchestrates and supervises the whole process in a quite controlled modest case study. Key elements in terms of organizational interoperability have been to get a common understanding of the procedures and the roles of each of the parties, including data access; and facing the challenges regarding the technical deployment of the infrastructure.

As for the first, it has been important to take the time to clarify the roles of the different actors in the federation. So, it has been needed to extensively explain the Coordination Hub, as the one orchestrating all the procedure from the development of the data model, the implementation of the analytical pipeline, the adaptation of the technological solutions to the computational environment of each node, acting as a help desk with the contact persons in each node, and the supervision of the process so as to synchronize the work in the different nodes. Most importantly, it has been necessary to explain the roles and requirements for the nodes as those that were basically in charge of providing information on the actual data access and ability to comply with the common data model and provide insight to adapt it to local specificities, effectively access to the required data, and above all set up a team in house that is able to 
comply with the requirements of the case study, particularly the deployment of the technological solutions.

As for data access challenges, the case study was designed to get the intended outputs with a very simple data model and rather limited data requirements. So, in this very controlled context, questions such as linkability of data sources, insufficient coverage or lack of relevance of the data sources, or more in depth data quality elements at variable level such as incompleteness, missingness or systematic errors have not been deemed as big challenges to deal with in this very exercise.

So, the main challenge in the deployment of the federation was expected to be (and it has been) the need for technical capacities in some of the nodes of the infrastructure, in particular, IT profiles that can easily implement or deploy the technological solutions developed by the Coordination Hub. An extra effort on supervision and capacity building has been assumed by the Coordination Hub that could not be sustainable in the eventual scaling up of the infrastructure.

\section{Challenges on semantic interoperability}

As in any cross-national comparative research, semantic (and syntactic) interoperability is the main challenge. Each of the entities composing the data model (patient, contact, event, time) are subject to threats to semantic (and syntactic) interoperability. As a matter of example, the definition of the cohort (how to define stroke and refine the strategy to select stroke patients); how specific or sensitive is the definition of ischemic or hemorrhagic stroke, the definition of episode, the identification of the care activities and where these activities are provided; and if these activities can be separate out across care providers, the uneven granularity of the timestamps, or how exiting the process is defined.

The effort made to get the data model common has implied understanding the care processes in the different nodes of the federation, agreeing on common concepts (and then, definitions) for the different attributes within entities, if there were different standards or encoding systems build the appropriate cross-walks, transforming the variables when needed to a common format or, in the worst scenario, reaching a minimum common denominator.

In this very exercise, the main threats to semantic (and syntactic) interoperability that the Coordination Hub had to solve have been:

- Reaching a consensus on the specification of the relevant activities to map in terms of acknowledging the hyper-acute care process in stroke (i.e. relevant therapies such as fibrinolysis or mechanical thrombectomy, but also CT scan image, etc.)

- Reaching a consensus on the classification of a stroke as ischaemic, transient ischemic or hemorrhagic using both ICD-9th and ICD-10th.

- Defining normalized dictionaries for certain concepts, such as 'discharge_code', based on mapping all existing categories in each partner with similar descriptors. 
- Setting a common default timestamp granularity and establishing the rules to comply with such granularity while being consistent with the care process, even when such level of granularity was not available at each site (i.e., requiring date-time granularity with 00:00:00 time when only date was available and checking the consistency of the timestamps on an expected sequence of activities to assess irregularities).

- Establishing a normalized file format (CSV file, comma separated, without quotation marks) and encoding (UTF-8) with preset headers and fixed variable name fitting the common data model specifications.

\section{Challenges on technological interoperability}

As stated previously, so as to guarantee full technical interoperability, the analysis scripts should be able to run independently of the available systems in the partners with all dependencies included within the containerized solution. Then Docker-based deployment relies on the availability of operating system level support for Docker containers among partners, commonly Linux-based servers.

However, some partners do not have Linux servers. For those cases the Coordination Hub implemented a set of virtual machine images containing Linux plus the Docker software, replicating the native environment previously described. These images were created in Open Virtualization Format version 2 [10] and Virtual Hard Drive version 2 format [11]; and can be deployed in virtually all commercial systems (Microsoft Windows, Apple macOS, multiple *NIX variants, etc.) and have demonstrated their utility during the project development. For the purposes of this case study the images used were adapted from the virtual machine images used in [12].

\section{Upcoming Challenges And Recommendations}

\section{On legal interoperability}

In the real life implementation of a federated approach, where many more nodes with different responsibility on the data curation and management, many more data sources could be used and data requirements could be larger in a context where research questions and queries grow exponentially. In this case, assuring the compliance with GDPR principles, in particular minimization and confidentiality, gains relevance and imposes new actions. In this context, Data Protection Officers (DPOs) will play a major role.

Recommendation 1: In the real life expansion of the JA Infact, data access will require to document how GDPR principles will be assured, mainly throughout: a) a protocol of the study behind the query (including the purpose and methodology) and a data management plan including the data schema (entities, variables, operational description with categories and values, and encoding systems), what are the measures to assure confidentiality and minimisation; who will be the actors and what role they will pay in data management and for how long.

\section{Recommendation 2}


In the context of the nodes, there will be a need for DPOs to understand how data accessing and data management procedures will work in the context of a federated approach. Specific training programs for DPOs could be recommendable. The other way round, the continuous exchange with DPOs will make each node be aware of the local and specific requirements and anticipate the data accessing needs.

\section{Recommendation 3}

In a scaled context, there will be a need for technological solutions that ensures security-and privacy-bydesign. Robust authentication and authorization systems will be needed to manage data access to only authorized users and to provide traceability information for forensic analyses in the follow up of a given user.

\section{On organizational interoperability}

In the JA InfAct case study the number of actors interacting has been confined to a few: in the Coordination Hub a technological and a domain expert, in the different nodes one or two contact persons with mixed profiles. In this very controlled case study, bilateral interaction between the coordination node and the four participan nodes, close tutoring of the process, even online remote intervention can be used to solve queries on the deployment of the technological solutions.

As mentioned when describing the federated research infrastructure, a good wealth of tasks are developed inhouse by each of nodes within the federation - discussing of the research question, agreeing on a common data model, accessing and collecting the data in the way required by such a data model, deploying the technologies developed elsewhere in their technological infrastructures, running the scripts, interpreting the error logs and the outputs.

In a federation with much more nodes, or in a hybrid federation with one node serving as an orchestrator of other nodes, or in a peer to peer federation where any node can orchestrate or any node can interrogate the federation the needs for organizational interoperability will sky rocketed.

Recommendation 4: In the context of a scaled up infrastructure, nodes in the federation will require a number of profiles: domain experts (depending on the research question), data scientists, data managers and system administrators. The Coordination Hub requires, in addition, an IT profile expert in distributed computing.

\section{Recommendation 5}

Orchestrating the whole distribution in more complex federations will require a stepwise approach (see details in Appendix) that smooths down the exchange between the Coordination Hub and the nodes, while deploying an analytical pipeline that is transparent and reproducible at any step.

\section{Recommendation 6}


In the institutions composing the federation, rating data curation institutions according to their procedures to get data up-to-date and high quality; agreeing on a common data quality framework (see for example in [13]); cataloguing their data sources in a way that is standard (e.g., DCAT [14]); providing information on interoperability standards and reusability; publishing clear procedures to get access to their data.

\section{On semantic interoperability}

Data requirements within InfAct JA case studies have been intendedly limited and, consequently, the number of data sources and the data types. Thus, getting a common data model has then been rather uncomplicated. A pan European federated infrastructure that is expected to interact with unlimited research questions will require consider multiple data sources and many more data types. Some of them may come from routine collections; for example, administrative or claims data as the ones used in the stroke case study, disease-specific registries, population-based registries, socioeconomic repertoires, electronic and medical health records, data from lab tests, data from imaging tests, etc. Some of them from ad hoc data collections, e.g., DNA sequences, tissues, data from wearables, samples of texts or data from social media.

In addition to the variety of data sources is also the heterogeneity of data in their very nature (at one end, administrative data; at the other end, natural language) but also heterogeneous in the encoding systems. For example the inclusion of new countries in the stroke use case may have required other disease encoding systems, such as NOMESCO [15], OPCS [16], Leistsungkatalog [17] or ACHI [18]. In case of using data from lab tests LOINC [19] should have been mapped out.

Recommendation 7. In the context of a scaled up infrastructure, it would be recommendable to map out and catalogue the most prevalent semantic interoperability standards. In that sense, future initiatives should link to standards developers and curators, for example, using SNOMED [20], the ontology of reference terms for medical conditions.

Recommendation 8. A pan european federated infrastructure should link with the existing research infrastructures on health data. On the one hand, to learn how they have catalogued the standards of semantic (and syntactic) interoperability. On the other hand, to provide access to their standards to the population health research community that could be interested in data models including that variety of data sources. As a matter of example, standards on biosamples [21] or omics [22].

Recommendation $9 \mathrm{It}$ is expected that most of the studies in an expanded version of a federated research infrastructure, the vast majority of research will be observational studies. A major multiparty initiative pursuing a common data model for observational research is OMOP. [23] A close follow up of this initiative is recommendable, even proactively advocating improvements to get the specificities of population health research well represented in the OMOP CDM.

\section{On technological interoperability}


Although setting up the foundations for the deployment of more complex pipelines the aforementioned technological elements in the JA InfAct stroke case study had a very modest scope. The eventual expansion of a federated research infrastructure such as the one tested in InfAct would require a technological upgrade considering three elements: the reduction of human interaction in the steps proposed in 4.2.2; the possibility of heavier computational workloads, and designing the architecture to allow full distribution of complex methodologies whose results are comparable to the ones using data pooling in a single repository.

The JA-InfAct federated analysis infrastructure can be considered a step towards more sophisticated solutions. It is a reliable solution for a problem specific scenario, but the foundations may be easily extended to include more analysis pipelines. For example, a generalized version of the infrastructure may support fully distributed statistical algorithms [24], [25] and, in the final term, state-of-the-art federated learning algorithms [26][27][28], the current cutting-edge analysis approach when leading with huge data sets distributed across multiple locations, without having the possibility of merging them. In addition, the current client-server architecture, which relies on a Coordination Hub that agglutinates a high level of responsibility can be moved to a peer-to-peer architecture, where all partners/Data Hubs can act as peers, having the capacity to coordinate analyses through the infrastructure.

Recommendation 10 When it comes to reducing human interaction, a way forward will be developing and implementing a robust deployment protocol to automatically orchestrate the federation set up activities between the Coordination Hub and the different, detailed in the stepwise process represented in Appendix.

Recommendation 11. One of the tasks of the coordination hub in an eventual scaled up federated infrastructure should be the assessment of the computational needs of the different research queries. To optimize the infrastructure resources and reduce the management overheads, it is sensible to outsource the computing or storage capabilities instead of having and maintaining high capacities inhouse. A solid European service providers such as, EGI, for computation capacities https://www.egi.eu, or EUDAT, for storage capacities (https://www.eudat.eu/) are primary choices for this purpose

Recommendation 12. In federated infrastructures, when research questions and research methodologies become more demanding the design of distributed analyses becomes paramount. These new and growing requirements should be faced in a future federated infrastructure by providing state-of-the-art federated machine learning algorithms and methods, as well as to provide the elements to easily develop or adapt new analysis techniques.

To conclude, it is important to note that all the know-how gathered during the development of the JAInfAct federated analysis infrastructure and some of the recommendations provided are currently being implemented in PHIRI, population health information research infrastructure [https://www.phiri.eu], a practical roll out of DIPOH, distributed infrastructure on population health research, current candidate to get into the ESFRI roadmap. In addition, all this insight is playing a fundamental part of the European Health Research and Innovation Cloud, a cloud for health data exchange between European health research infrastructures and health services, to be designed under the HealthyCloud project 
[https://healthycloud.eu]. Finally, this knowledge is currently helping to give shape to the future European Health Data Space, the project to regulate the secondary use of health data across Europe, under the framework of the TEHDaS Joint Action [https://tehdas.eu].

\section{Conclusion}

The JA InfAct federated analysis infrastructure has been able to cope with all levels of the interoperability -legal, organisational semantic and technological. Any proposal for extension would require upgrade so as to deal with new challenges on interoperability such as large scale application of GDPR principles, developing high-profile resources capacity across Europe, developing a common strategy to data quality assurance and preparedness for high performance computing and federated learning.

\section{Abbreviations}

DPO Data Protection Officers

EIF European Interoperability Framework

ETL Extraction Transformation and Load

FAIR Findable, accessible, interoperable, reusable

FRI Federated research infrastructure

GDPR General Data Protection Regulation

CDM Common Data Model

CSV Comma-separated values

CT Computed tomography

ICD International classification of diseases

ICT Information and communication technologies

IT Information technologies

*NIX Operative system similar to UNIX

PC EHR Primary Care Electronic Health Records

SQL Structured query language

\section{Declarations}




\section{Ethics approval and consent to participate}

This study, observational in design, used retrospective pseudonymized, non-identifiable and non-traceable data, and was conducted in accordance with the amended Helsinki Declaration, the International Guidelines for Ethical Review of Epidemiological Studies, and European legislation on data protection and patients' rights. The study implies the use of pseudonymised data, accesses and/or curated and/or treated under the ethical and legal provisions of application in the different participant institutions. The work has been developed in the context of a federated infrastructure where no individual data is pooled or exchanged across institutions but just aggregated outputs.

\section{Consent for publication}

Not applicable

\section{Availability of data and material}

This research builds on a federated research approach where data holders keep the data in their premises (ie, no individual nor aggregated is pooled centrally) but only scripts and aggregated results move. All scripts have been developed on open source solutions, transparent to be audited, and available under Creative Commons Attribution 4.0 International licensing.

\section{Competing interests}

The content of this report represents the views of the authors only and is their sole responsibility; it cannot be considered to reflect the views of the European Commission and/or the Consumers, Health, Agriculture and Food Executive Agency (CHAFEA) or any other body of the European Union. The European Commission and the Agency do not accept any responsibility for use that may be made of the information it contains.

\section{Funding}

Joint Action InfAct. Third Health Programme Grant \#801553

\section{Authors' contributions}

JGG, FER, CTO and EBD have participated in the conceptual design of the study. JGG, CTO and JGGa have developed and implemented the technological elements for the deployment of the JA Infact federated infrastructure. FER, JGGa, LP, AF, IP, JV, JM and IZ have contributed data, and have deployed the federated infrastructure in their premises, have run the analysis and interpreted the results. EBD and JGG are main contributors in drafting the final version of the paper. All the authors have reviewed and approved the submitted document.

\section{Acknowledgments}


The present work has benefited from the continuous exchange with all the participant researcher in the Joint Action InfAct

\section{Joint Action InfAct Consortium contributors}

Brigid Unim ${ }^{3}$, Flavia Carle ${ }^{7}$, Rosaria Gesuita ${ }^{7}$,Damir Ivanković ${ }^{5}$, Marko Brkić $^{5}$, Jelena Dimnjaković ${ }^{5}$, Jane Lyons $^{8}$, Ronan Lyons ${ }^{8}$, Zeynep Ors ${ }^{9}$, Metka Zaletel ${ }^{10}$, Paulo Nogueira ${ }^{11}$, Luís Velez Lapão ${ }^{12}$, Håkon Haaheim $^{13}$, Petronille Bogaert ${ }^{14}$, Linda Abboud ${ }^{14}$, Herman van Oyen ${ }^{14}$

1. Università Politecnica delle Marche, Italy

2. University of Swansea, Wales, UK

3. IRDRES, Paris, France

4. National Institute of Public Health, Ljubljana, Slovenia

5. Ministério da Saúde, Lisboa, Portugal

6. Instituto de Higiene e Medicina Tropical - Universidade Nova de Lisboa, Lisboa, Portugal

7. Directorate of Health, Oslo, Norway

8. Sciensano, Brussels, Belgium

\section{References}

1. Directorate-General for Informatics (European Commission). New European Interoperability Framework. Brussels Nov. 2017. doi:10.2799/78681.

2. Gonzalez-Garcia J, Telleria-Orriols C, Estupinan-Romero F, Bernal-Delgado E, "Construction of Empirical Care Pathways Process Models From Multiple Real-World Datasets," IEEE J. Biomed. Heal. Informatics, vol. 24, no. 9, pp. 2671-2680, Sep. 2020, doi: 10.1109/JBHI.2020.2971146.

3. van der Aalst WMP. Process Mining: Data Science in Action. Second ed. Berlin: Springer Berlin Heidelberg; 2016.

4. Mans RS, van der Aalst WMP, Vanwersch RJB. Process Mining in Healthcare: Evaluating and Exploiting Operational Healthcare Processes. Cham: Springer International Publishing; 2015.

5. Gomez CR, Malkoff MD, Sauer CM, Tulyapronchote R, Burch CM, Banet GA. Code Stroke. An attempt to shorten in hospital therapeutic delays. Stroke. 1994;25(10):1920-3. doi:10.1161/01.STR.25.10.1920.

6. European Parliament and Council of the European Union. Regulation (EU) 2016/679 of the European Parliament and of the Council of 27 April 2016 on the protection of natural persons with regard to the processing of personal data and on the free movement of such data, and repealing Directive 95/46/EC (General Data Protection Regulation). Brussels: European Parliament and Council of the European Union, 2016.

7. World Health Organization. International classification of diseases: [9th] ninth revision, basic tabulation list with alphabetic index. World Health Organization; 1978. 
8. World Health Organization. International statistical classification of diseases and related health problems. 10th Revision, 5th ed. Geneva, 2015.

9. Boettiger C, "An introduction to Docker for reproducible research," in Operating Systems Review (ACM), Jan. 2015, vol. 49, no. 1, pp. 71-79, doi: 10.1145/2723872.2723882.

10. Distributed Management Task Force Inc., Open Virtualization Format Specification (DSP0243). 2015.

11. Microsoft, Corporation, Virtual Hard Disk v2 (VHDX) File Format, 5th Revision. 2021.

12. González-García J, Tellería-Orriols E-RF, Carlos, \& Bernal-Delgado E. (2020, July 20). Stroke Process Mining Analysis Virtual Machine Image (OVFv2 / VHD formats) (Version Data Model Slides v13 (Effective Data Model v11). Run Slides v7.). Zenodo. http://doi.org/10.5281/zenodo.3952495.

13. Health Data Research UK Data Quality and Standards Strategy Green Paper for Consultation September. 2019; available at: https://ukhealthdata.org/wpcontent/uploads/2019/09/HDRUK_Data_Quality_Standards_Green_Paper_Sept2019circulate.pdf (last access, 2021, 31st May).

14. DCAT Data Application Profile for Data Portal in Europe, European Commission. 2021, available at https://ec.europa.eu/isa2/solutions/dcat-application-profile-data-portals-europe_en (last access, 2021, 31st of May).

15. N.-N. Nordic Council of Ministers, NOMESCO Classification of Surgical Procedures (NCSP), Version 1.14. Copenhagen, 2009.

16. National Clinical Coding Standards OPCS. Terminology and classifications delivery service. NHS Digital. 2021. Available at: https://classbrowser.nhs.uk/ref_books/OPCS-4.9_NCCS-2021.pdf (last access, 2021, 31st May).

17. Leistsungkatalog MH, Austria. 2021. Available at https://www.sozialministerium.at/public.html (last access, 2021, 31st May).

18. ICD-10-AM/ACHI/ACS IHAP. 2021. Available at https://www.ihpa.gov.au/publications/icd-10amachiacs-ninth-edition (last access, 2021, 31st May).

19. Common LOINC, Laboratory Observation Codes LOINC. 2021. Available at https://loinc.org/usage/obs/ (last access, 2021, 31st May).

20. Spackman KA, Campbell KE, Côté RA. "SNOMED RT: A Reference Terminology for Health Care," J. Am. Med. Informatics Assoc., vol. 4, no. SUPPL., pp. 640-644, 1997, Accessed: Apr. 27, 2021. [Online]. Available: /pmc/articles/PMC2233423/?report = abstract.

21. BBMRI-ERIC standardization services Available. at https://www.bbmrieric.eu/services/standardisation/ (last access, 2021, 31st May).

22. ELIXIR-ERIC interoperability and standards. Available at https://elixireurope.org/services/tag/interoperability-and-standards (last access, 2021, 31st May).

23. OHDSI, Observational data sciences and informatics. 2021. Available at https://ohdsi.github.io/TheBookOfOhdsi/ (last access, 2021, 31st May). 
24. Shi P, Wang P, Zhang H, "Distributed Logistic Regression for Separated Massive Data," in Communications in Computer and Information Science, Sep. 2019, vol. 1120 CCIS, pp. 285-296, doi: 10.1007/978-981-15-1899-7_20.

25. Lu C-L, et al., "WebDISCO: a web service for distributed cox model learning without patient-level data sharing," J. Am. Med. Informatics Assoc., vol. 22, no. 6, pp. 1212-1219, Nov. 2015, doi: 10.1093/jamia/ocv083.

26. Yang Q, Liu Y, Chen T, Tong Y. Federated machine learning: Concept and applications. ACM Trans Intell Syst Technol. Jan. 2019;10(2):1-19. doi:10.1145/3298981.

27. Bonawitz K, et al., “Towards Federated Learning at Scale: System Design,” 2019, [Online]. Available: https://arxiv.org/abs/1902.01046.

28. Li T, Sahu AK, Talwalkar A, Smith V. Federated Learning: Challenges, Methods, and Future Directions. IEEE Signal Process Mag. May 2020;37(3):50-60. doi:10.1109/MSP.2020.2975749.

\section{Figures}

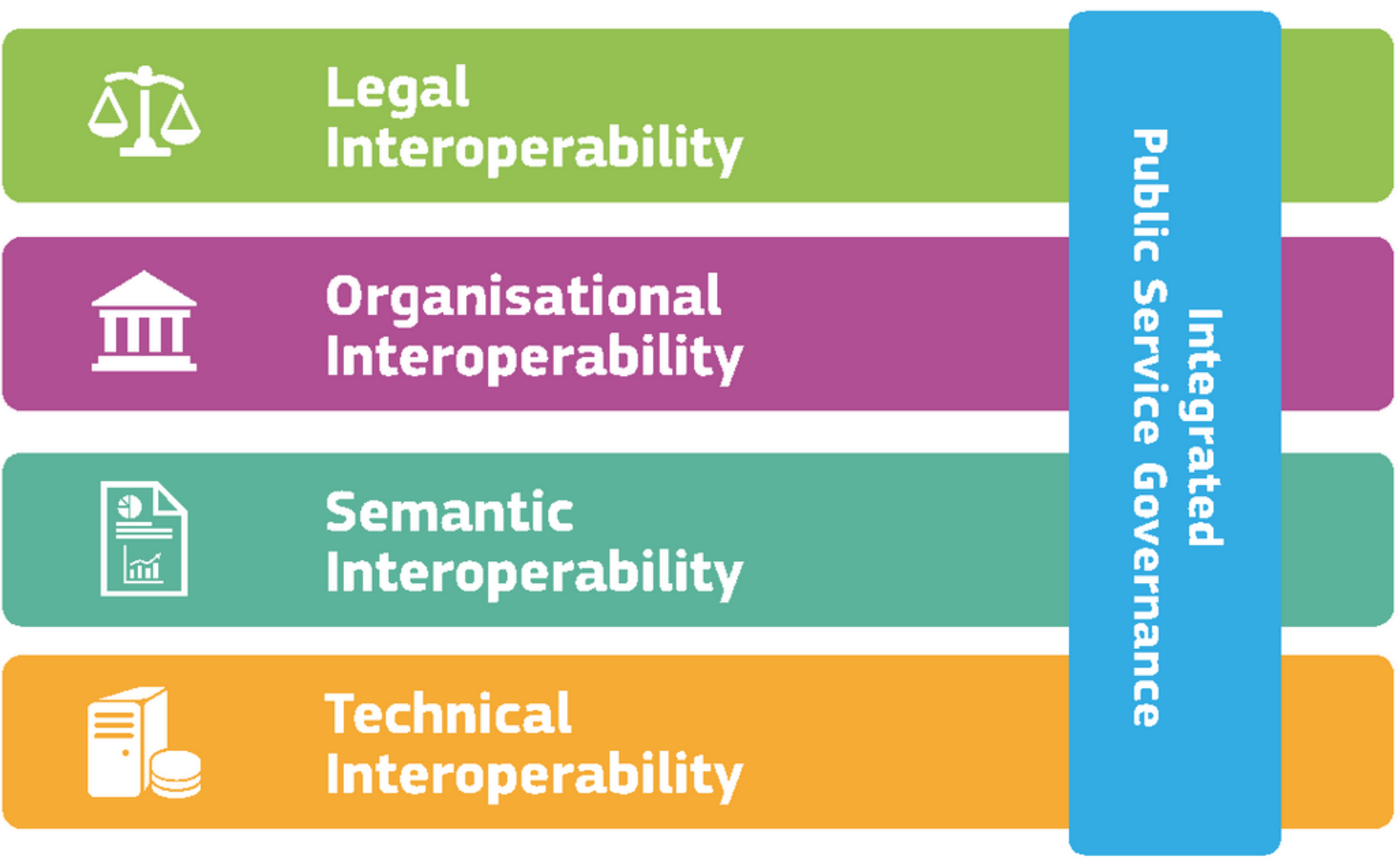

\section{Figure 1}

European Interoperability Framework interoperability model. 


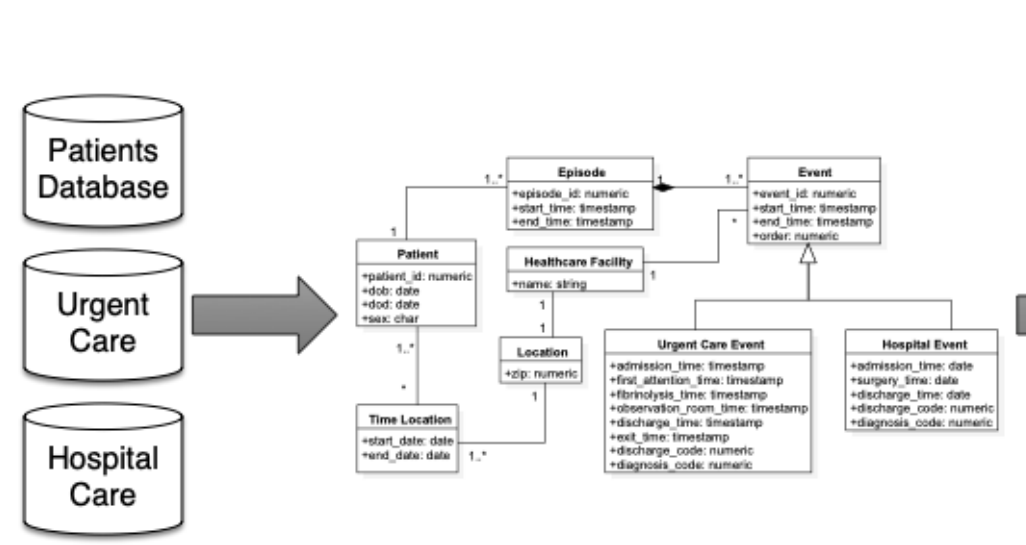

Real-World Datasets
Common Data Model
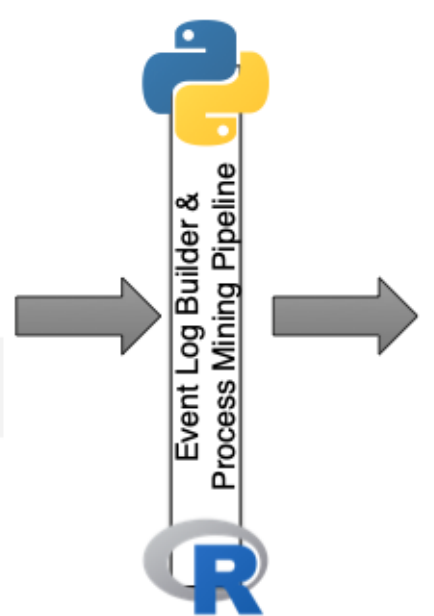

Data Mining



Process Mining Models

\section{Figure 2}

Methodology and analytical pipeline supporting the case study on stroke

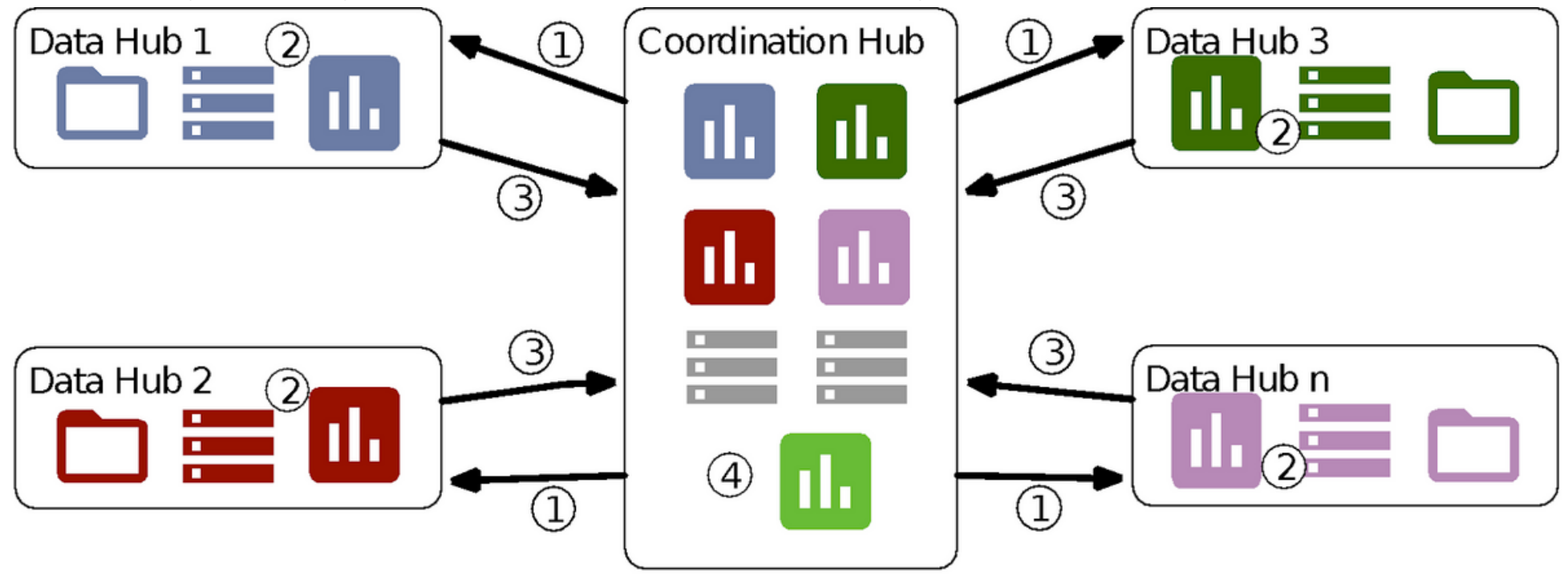

Figure 3

JA-InfAct federated analysis infrastructure 


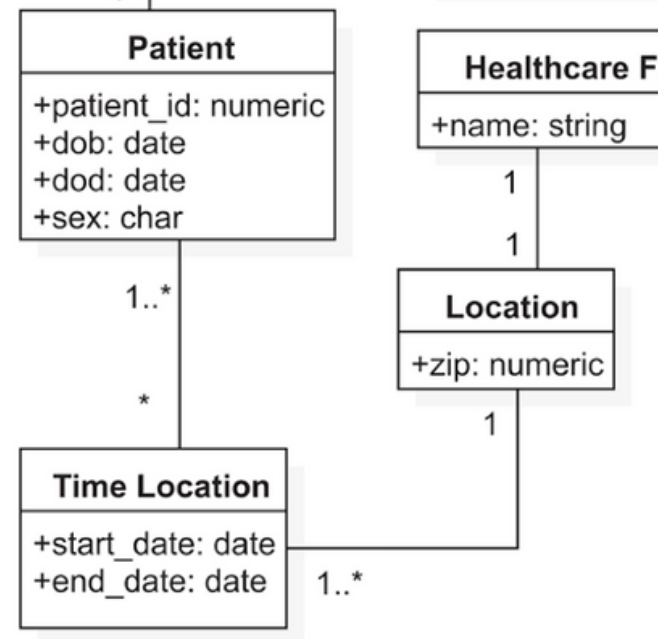

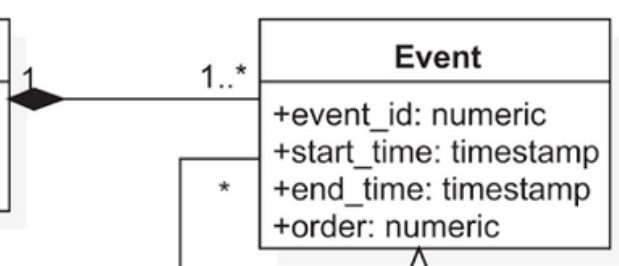

order. numeric

\section{Figure 4}

Stroke care data model - Conceptual diagram. 


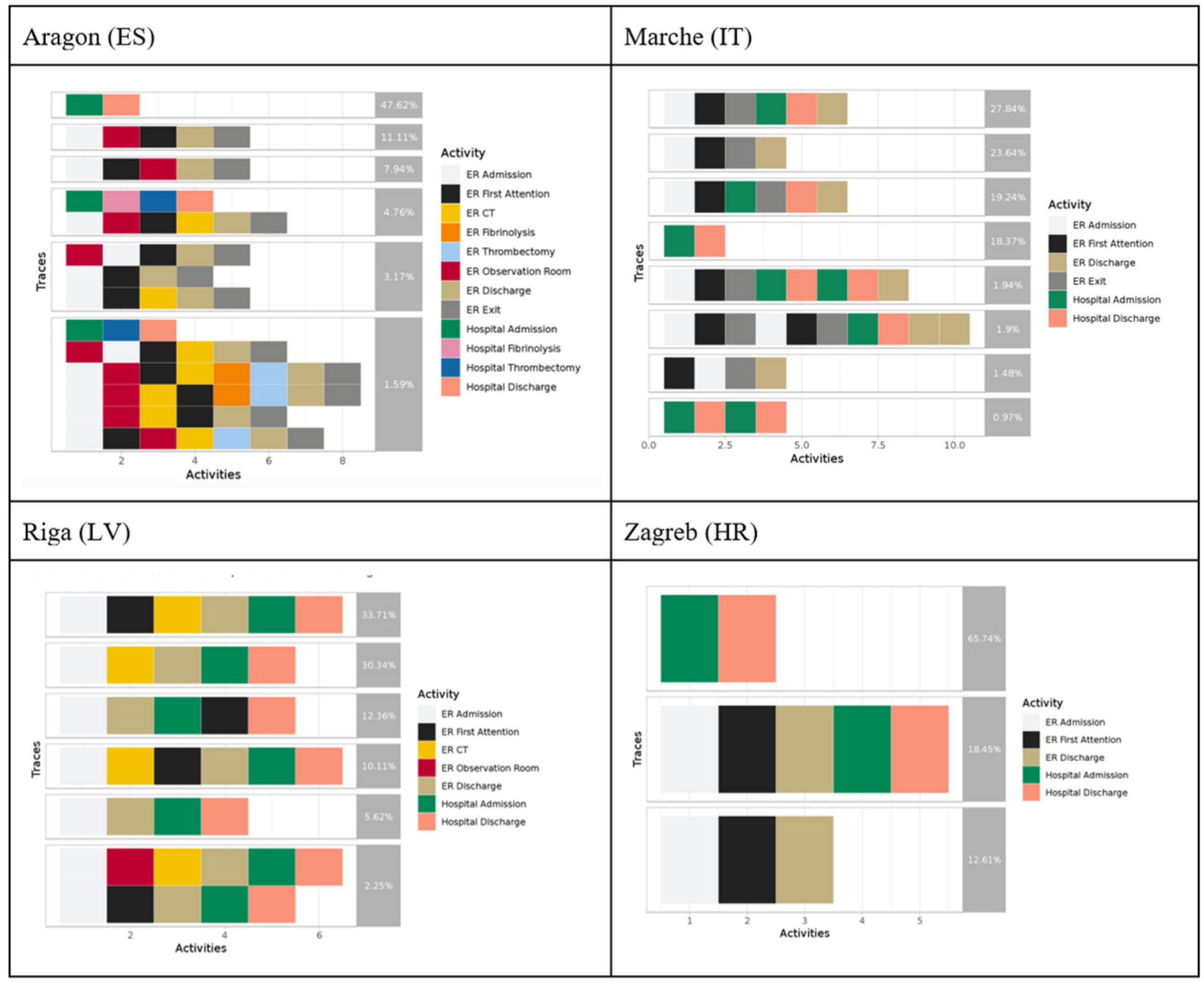

Figure 5

Process traces 


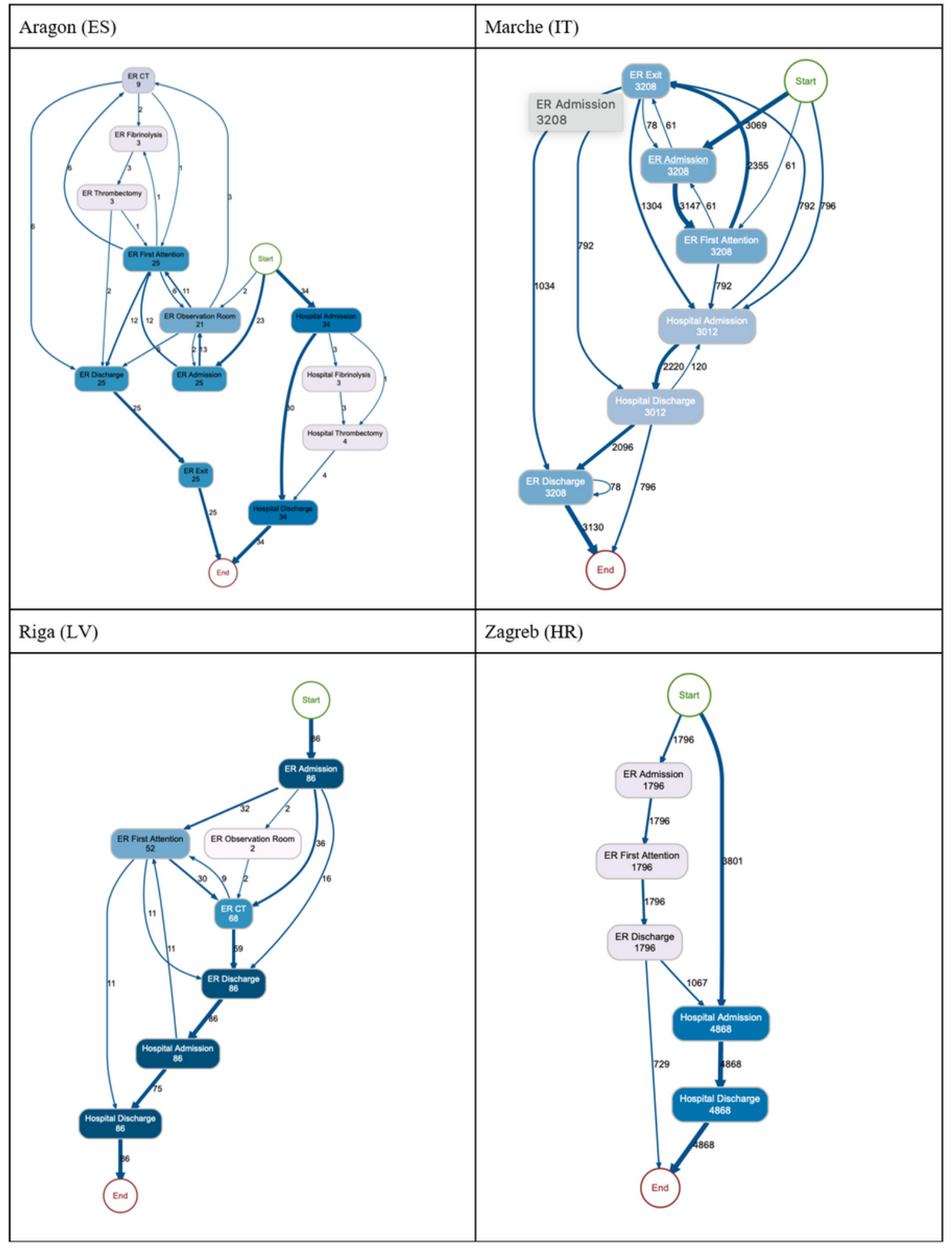

Figure 6

Number of patients across the pathway 


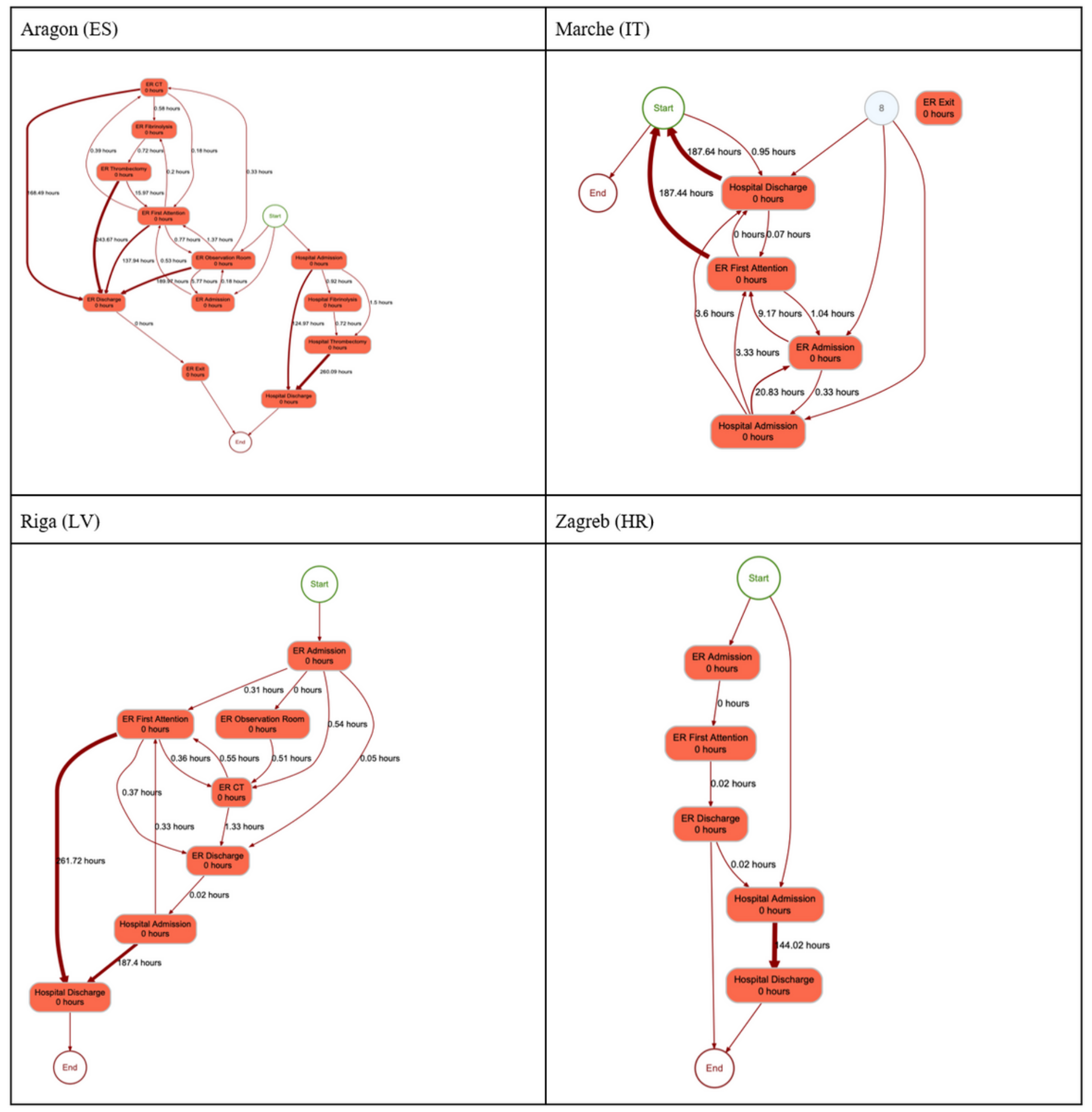

Figure 7

Throughput times 




Figure 8

Matrix of precedence

\section{Supplementary Files}

This is a list of supplementary files associated with this preprint. Click to download.

- Appendix.docx 